\title{
A Rare Case of Bilateral Benign Ovarian Tumours with Asymptomatic Bicornuate Uterus in a Postmenopausal Woman.
}

\author{
Dr. Kakarla Jaya Prada Devi ${ }^{1}$, Associate Professor, Dr. Adusumilli Padmaja ${ }^{2}$, \\ Assistant Professor, Dr. Prashanthi Vemulapall ${ }^{3}$, Assistant. Professor, \\ Dr. V. Geetha Sree ${ }^{4}$, Post Graduate \\ 1, 2, 3,4 (Dept. of OBG, Dr. PSIMS \& RF, Chinoutpalli, AP, India)
}

\begin{abstract}
The incidence of postmenopausal ovarian tumours range to $3-18 \% *^{1}$. Most of the ovarian tumours in postmenopausal age group are taken as malignant unless proved otherwise, more so when they are bilateral $*^{2}$. We are reporting a case of benign bilateral ovarian tumour in a postmenopausal lady. Incidentally the woman also had a bicornuate uterus with a rudimentary horn. The incidence of mullerian anomalies range to 2- 3\%**. They are known to cause pregnancy losses, preterm labour, labour dystocia $*^{4}$ \& gynaecological symptoms like dysmenorrhoea and AUB. The above mentioned patient had uneventful obstetric history therefore emphasizing that mullerain anamolies do not need intervention when they are asymptomatic ${ }^{* 5}$.
\end{abstract}

Key words: Bilateral ovarian tumours, Bicornuate uterus, postmenopausal.

\section{Introduction}

The incidence of postmenopausal ovarian tumours range to 3-18\%. Ovarian tumour in a postmenopausal women, more so when they are bilateral turn out to be malignant. The incidence of mullerian anomalies range to $2-3 \%$. They are known to cause early miscarriages, preterm labour \& labour dystocia and gynaecological symptoms like dysmenorrhoea \& AUB, however some of them may be asymptomatic and picked up incidentally.

\section{Case Report}

Mrs X aged 54 yrs postmenopausal lady reported to Gynaec OPD at Dr. PSIMS \& RF with complaints of lower abdominal pain and distention of $4-5$ months duration. She is P4L4 all uneventful home deliveries attained menopause 6 yrs back. There was no history of weight loss or loss of appetite or altered bowel and bladder habits or postmenopausal bleeding. On examination patient was moderately built \& nourished with BMI 20.6. Patient was pale, vital stable, CVS \& RS - NAD. P/A - central obesity present, soft no organomegaly, no tenderness, Vulvovaginal examination was normal. P/S - cervix and vagina healthy. Bimanual examination, uterus retroverted normal size, cystic mass was palpated through both lateral fornices, size could not delinated due to central obesity. Mass was not mobile, nontender. PR rectal mucosa was free. Patient was investigated, HB: 8 gm\%. Pap smear was done. No abnormality detected.

USG : Shows uterus size $6.1 \times 3.8 \times 3.1 \mathrm{~cm}$ size echopatten normal endometrium thickness $3 \mathrm{~mm}$. A large complex cyst measuring $12.2 \times 10.5 \times 9.5 \mathrm{~cm}$ with thick septations and solid components involving pelvis superior to uterus and urinary bladder. Both ovaries were not separately visualized. CA 125: 3 IU. After correction of anaemia patient was posted for laparotomy.

Intraoperative findings : No asictes, omentum was normal, uterus was bicornuate with a rudimentary right horn $2 \times 2 \mathrm{cms}$ as shown in the pic 1 . The rudimentary horn was connected to the uterus by a thick fibrous band at the level of cervix.

Both the ovaries were enlarged right side measuring $5 \times 4 \mathrm{~cm}$ and left side measuring $5 \times 3 \mathrm{~cm}$. A cyst measuring $8 \times 8 \mathrm{~cm}$, surface was smooth cystic consistency arising from the left ovary was present. On the right side a cyst of $12 \times 10 \mathrm{~cm}$ with bosselated surface, arising from the right ovary was seen. Cut section revealed multi cystic areas with clear fluid with solid area as shown in pic 2 . Both ovaries with cystic masses were sent for frozen section. Which revealed serous cyst adenoma. We proceeded with TAH + BSO. Patient recovered well postoperatively.

Histopathology report: Bicornuate uterus with bilateral papillary serous cyst adenofibroma. 


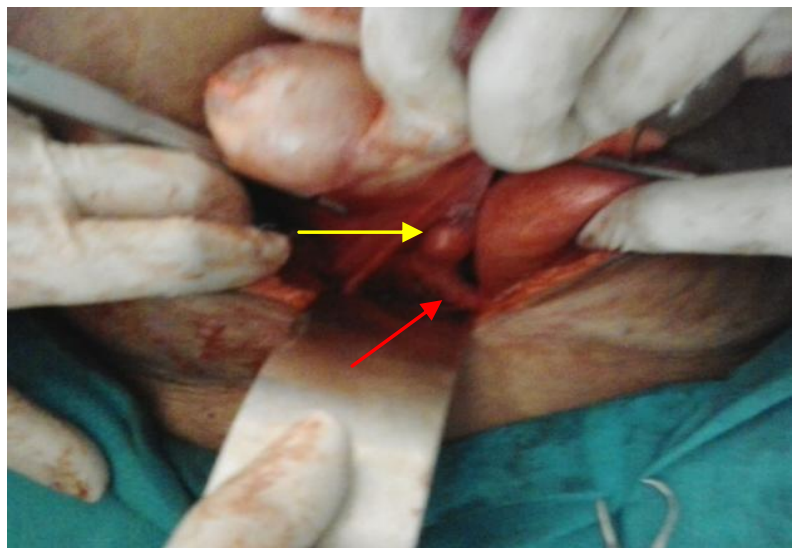

Picture 1: Bicornaute uterus with rudimentary right horn (yellow arrow), fibrous band connecting uterus to rudimentary horn (red arrow).

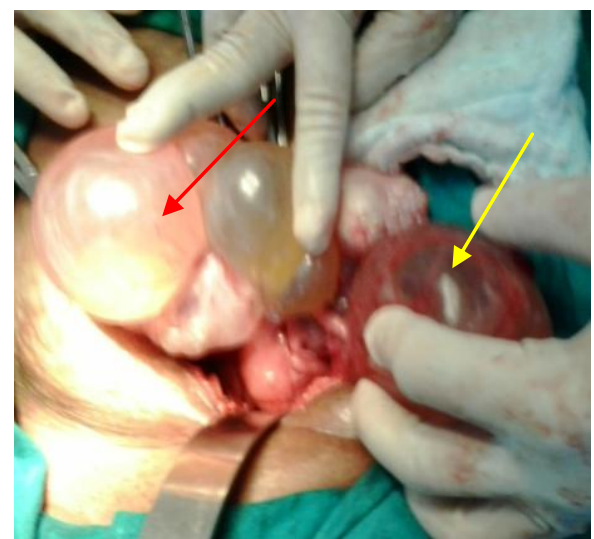

Picture :2 showing right side ovarian cyst (yellow arrow), left side ovarian cyst (pink arrow).

\section{Discussion}

Postmenopausal ovarian masses should be considered as malignant unless proved otherwise. Bilaterality of tumour favours more towards malignancy. CA 125 is one of the tumour marker for epithelial ovarian markers but negative CA 125 does not rule out malignancy. In the above case CT scan was not done as the patient was not affordable and also examination \& history was not favouring towards malignancy. But CT scan is also a valuable investigation in case of ovarian malignancy. There fore all postmenopausal ovarian masses should be evaluated thoroughly. Incidentally the patient had a bicornuate uterus with rudimentary right horn. It is well known fact mullerian anomalies can present with several symptoms ranging from subfertility to pregnancy losses, preterm labour, labour dystocia \& gynaceological symptoms like dysmenorrhoea \& AUB. The above mentioned patient had four uneventful home deliveries \& no menstrual symptoms, emphasing that asymptomatic mullerian anamolies diagnosed incidentally in any age group should not rise a concern \& treatment should be aimed only when they are symptomatic.

\section{Acknowledgements}

Staff members, Dept of OBGYN, Dr. PSIMS \& RF

Dept of Radiology, Dr. PSIMS \& RF

Dept. of Pathology, Dr.PSIMS \& RF

\section{References}

[1]. Ovarian tumour in postmenopausal woman, The Canadian journal of CME/Dec2007.

[2]. Hoskins WJ. Principles and practice of gynecologic oncology, 4th ed. Philadelphia, PA: Lippincott Williams \& Wilkins, 2005:xix, 1419

[3]. Mullerian duct anomalies : Clinical concepts Elyan A, MD and Saced M, MD. ASJOG VO1 1, Jan 2004.

[4]. Rackow BW, Arici A (2007) “ Reproductive performance of women with mullerian anomalies” curr. Opin. Obset. Gynecol.19 (3):229 -37 .

[5]. Junqueira BL, Allen LM, Spitzer RF et-al. Müllerian duct anomalies and mimics in children and adolescents: correlative intraoperative assessment with clinical imaging. Radiographics. 29 (4): 1085-103. 